\title{
Management of Symptomatic Intraluminal Thrombus of The Internal Carotid Artery
}

\section{Introduction}

Intraluminal thrombus of the internal carotid artery (IT-ICA) is an infrequent finding in acute ischemic stroke (AIS)

Nonocclusive thrombi are often found incidentally on angiographic studies increasing the risk of recurrent AIS and Large Vessel Occlusion

Treatment varies, and guidelines have yet to be established. We aimed to describe the variation in the treatments and evaluate for differences in recurrent stroke and intracerebral hemorrhage across treatment groups

\section{Methods}

A database of 1485 consecutive CTa, MRA and DSA was created retrospectively between September 1, 2011, and December 1, 2016

Radiology reports were queried for keywords including intraluminal, floating, clot, thrombus or filling defect. We identified cases of intraluminal thrombus of the extracranial ICA in patients over the age of 18.

Demographics, clinical features, and imaging characteristics were reviewed

\section{Results}

Of 84 radiology reports with intraluminal thrombus

- 37 patients (44\%) had IT-ICA and AIS

- Mean age 59 years; (IOR) $46.5-71.5$

- Median NIH Stroke Scale was 10.5 (IQR 1 - 9)

- $8(21.6 \%)$ patients received IV-tPA alone

- $10(27 \%)$ had LVO and underwent mechanical thrombectomy.

- Severe ICA stenosis (By NASCET criteria) was observed in 17 (46\%) patients

- $11(29.7 \%)$ had CEA

- 3 (8\%) ICA stenting.

Modified Rankin Scale

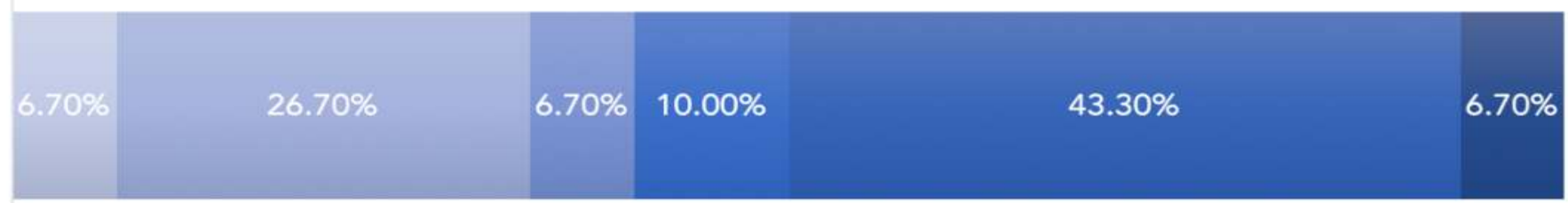

$\begin{array}{lllllllllll}0 \% & 10 \% & 20 \% & 30 \% & 40 \% & 50 \% & 60 \% & 70 \% & 80 \% & 90 \% & 100 \%\end{array}$ $\square 0=1=2 \square 3=4 \square 5 \square 6$

Modified Rankin Scale

$\mathrm{ICH}(n=6)$
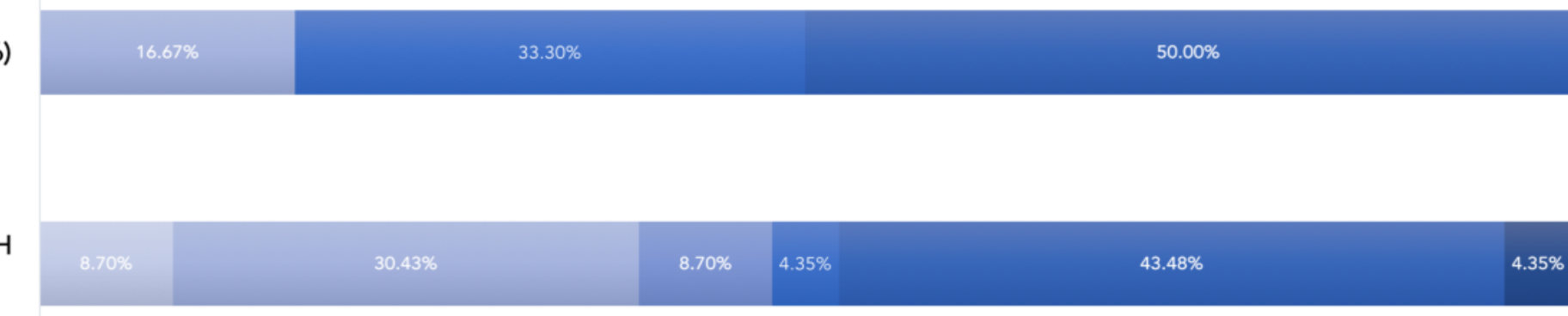

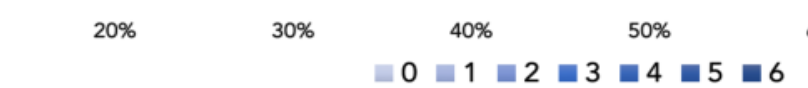

\section{Conclusions}

Most patients with IT-ICA and AIS were treated with anticoagulation and antiplatelet therapy

More than $10 \%$ of patients had recurrent AIS, and $16 \%$ had complications of $\mathrm{ICH}$.

The best treatment strategy to prevent recurrent AIS and minimize hemorrhagic transformation remains unclear.

Multicenter prospective studies are needed to evaluate optimal timing of CEA and safety and efficacy of medical management.
Results

Male, $\mathrm{n}(\%)$

$23(62.2 \%)$

Age, (IQR)

$59.4(46.5-71.5)$
$27(73.0 \%)$

$1(2.7 \%)$

$9(24.3 \%)$,

$1,(2.7 \%)$

NIHSS, baseline mean (IQR)

$10.5(1-9)$

\section{Risk Factors}

Hypertension n (\%)

$26,(70.3 \%)$

Hyperlipidemia $\mathrm{n}(\%)$

$12,(32.4 \%)$

Diabetes n (\%)

6, (16.2\%)

Smoking $n(\%)$

$13,(35.1 \%)$

\section{Degree of Stenosis}

Mild

9, (24.3\%)

Moderate

$7,(19.4 \%)$

Severe

$17,(45.9 \%)$

IV tPA administered $\mathrm{n}(\%)$
Mechanical Thrombectomy
IA tPA
CEA
CAS

IV tPA administered $\mathrm{n}$ (\%)

$8(21.6 \%)$

$10(27 \%)$

$2(5.4 \%)$

$11(29.7 \%)$

$3(8.1 \%)$

\section{Medications}

Aspirin $n$ (\%)

Clopidogrel n (\%)

Warfarin n (\%)

Heparin n (\%)

\begin{tabular}{rr} 
Home treatment & In Hospital \\
$7(18.9 \%)$ & $30(81.1 \%)$ \\
$2(5.4 \%)$ & $12(32.4 \%)$ \\
$4(10.8 \%)$ & $16(43.2 \%)$ \\
0 & $28(75.7 \%)$ \\
0 & $8(21.6 \%)$ \\
\hline
\end{tabular}

- Four patients had recurrent AIS during hospitalization

- Of the 8 patients who developed $\mathrm{ICH}$

- 6 were treated with anticoagulation

- 6 (16.2\%) did have a carotid intervention

- There was no statistically significant difference in the rate of $\mathrm{ICH}$ or recurrent AIS between the treatment groups.

\section{References}

Vellimana AK, et al. Symptomatic Patients with Intraluminal Carotid Thrombus: Outcome with a Strategy of Initial Anticoagulation. Journal of neurosurgery. 2013;118(1):10.3171/2012.9.

- $\quad$ Elijovich L, Mainali S, Doss V, Arthur A, Watridge C. Medical management of free-floating carotid thrombus.Clinical Neurology and Neurosurgery. 2013 Aug;115(8):1532-5

Ferrero $\mathrm{E}$, Ferri $\mathrm{M}$, et al. Free-floating thrombus in the internal carotid artery: diagnosis and treatment of 16 cases in a single center. Annals of Vascular Surgery. 2011 Aug;25(6):805-12 